\title{
Caracterização e uso da cinza do bagaço de cana-de-açúcar em tijolos de solo-cimento
}

\author{
Characterization and use of sugarcane bagasse ash in soil- \\ cement bricks
}

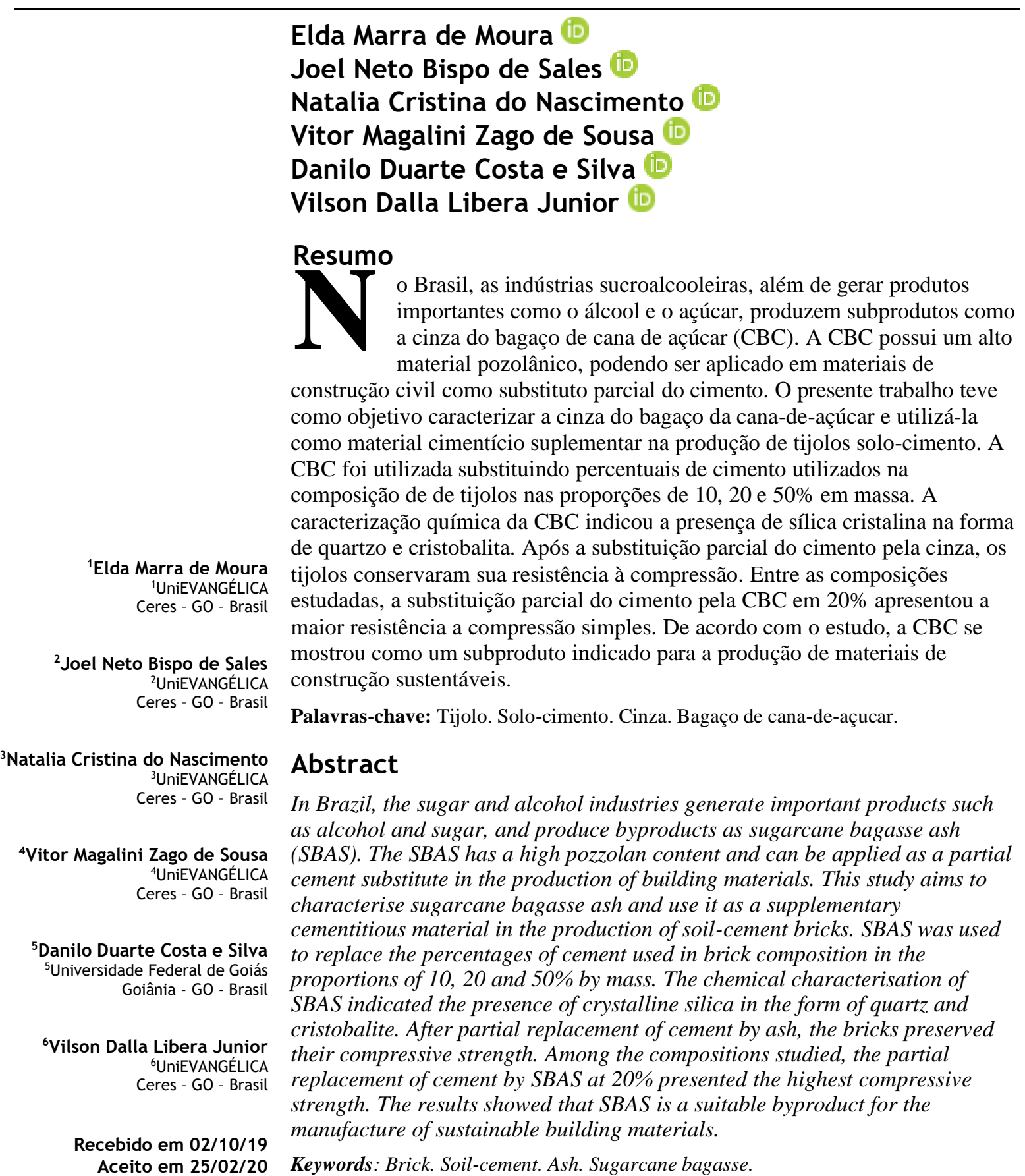

MOURA, E. M. de; SALES, J. N. B. de; NASCIMENTO, N. C. do; SOUSA, V. M. Z. de; COSTA E SILVA, D. D.; 


\section{Introdução}

Os materiais de construção (aço, concreto, tijolos queimados, etc.) são considerados materiais com altos níveis de carbono incorporado devido ao seu processo de fabricação originar a liberação de grandes volumes de gases de efeito estufa na atmosfera (TRAN; SATOMI; TAKAHASHI, 2018). Atualmente, o custo dos materiais de construção convencionais tem aumentado devido à energia necessária para a produção, contribuindo para a escassez de recursos naturais e o alto custo de transporte entre as fábricas e o canteiro de obras. Essas preocupações econômicas e ambientais têm gerado cada vez mais interesse em pesquisas que envolvam materiais de construção alternativos, como tijolos de solo-cimento e técnicas de construção mais sustentáveis (DANSO et al., 2015).

Os tijolos de solo-cimento têm se destacado como uma opção atraente para futuras construções. Estes tijolos apresentam baixo custo de produção e são considerados ambientalmente amigáveis, visto que, em seu processo de fabricação, não se emprega a queima, em comparação com os tijolos cerâmicos convencionais (RODRIGUES; HOLANDA, 2015). Os tijolos de solo-cimento são fabricados a partir da mistura em proporções adequadas de solo pulverizado, cimento e água. A adição de cimento ao solo contribui significativamente para o aumento de sua resistência mecânica, tornando o material final estruturalmente resistente, estável e durável (KOLLING; TROGELLO; MODOLO, 2012). Apesar do solo ser o elemento com maior proporção nos tijolos, a quantidade de cimento utilizada para estabiliza-lo ainda é considerada alta.

A produção de cimento contribui significativamente para a emissão de gases do efeito estufa através da liberação de grandes volumes de dióxido de carbono $\left(\mathrm{CO}_{2}\right)$. Estima-se que a produção de 1 tonelada de Cimento Portland libere na atmosfera 1 tonelada de $\mathrm{CO}_{2}$ (SCHNEIDER et al., 2011). Consequentemente, uma forma bem-sucedido de reduzir o impacto ambiental relacionado ao uso do cimento constitui na sua substituição parcial por materiais cimentícios suplementares (MCS), muitas vezes provenientes de indústrias ou resíduos (CHANDRA PAUL et al., 2019). Além de possuírem características sustentáveis, dependendo da sua atividade pozolânica, os MCS podem melhorar a resistência e a durabilidade dos materiais que utilizam o cimento como aglomerante (ARIF; CLARK; LAKE, 2016).

Entre os diversos tipos de MCSs, os resíduos industriais são frequentemente utilizados como pozolanas, incluindo cinzas volantes de carvão, sílica ativa e resíduos agrícolas como cinzas de casca de arroz e cinza de bagaço-de-cana de açúcar (CBC) (FRÍAS; VILLAR; SAVASTANO, 2011). A CBC é resultado da queima do bagaço de cana para geração do calor e energia utilizados em uma usina de açúcar. O Brasil, o maior produtor de cana-de-açúcar do mundo, gera mais de 2,5 milhões de toneladas de CBC por ano (FARIA; GURGEL; HOLANDA, 2012). Visando o aproveitamento deste material pós-processamento, diversas pesquisas têm sido conduzidas sobre a utilização de resíduos de CBC na indústria de construção civil, onde a maior parte dos trabalhos se concentra na sua aplicação como um substituto de cimento (CHANDRA PAUL, et al., 2019; FARIA; GURGEL; HOLANDA, 2012).

A CBC é considerada como um material pozolânico, obtido como subproduto da indústria sucroalcooleira. A CBC contém sílica amorfa e apresenta boas propriedades pozolânicas (MARTIRENA, et al., 1998; KANTIRANIS, 2004). As pozolanas tem capacidade de reagir com o hidróxido de cálcio liberado durante o processo de hidratação do cimento, formando compostos estáveis de poder aglomerante, tais como os silicatos e aluminatos de cálcio hidratados (OLIVEIRA et al., 2004). Entretanto, os diferentes processos de queima e coleta afetam as propriedades físicas e químicas da cinza.

Segundo Kazmi et al. (2016), a adição de cinzas da casca de arroz e do bagaço de cana-de-açúcar em tijolos de solo reduz o peso do material final e resulta na diminuição de cargas estruturais da edificação, contudo, seu estudo constatou-se que a resistência à compressão e o módulo de ruptura diminuíram de acordo com a adição desses materiais. Faria, Gurgel e Holanda (2012) analisaram o uso de CBC como matéria-prima na fabricação de tijolos de argila, onde os resultados demonstraram que a CBC pode ser usada como enchimento em tijolos, resultando em uma construção mais sustentável e econômica. Valenciano e Freire (2004) avaliaram as características físicas e mecânicas de misturas de solo, cimento e CBC. Diferentes combinações cimento-cinza foram analisadas e a resistência à compressão foi medida. Os resultados indicaram a possibilidade de substituir até $20 \%$ do cimento por CBC sem diminuir a resistência à compressão do material.

O presente trabalho teve como objetivo avaliar as características da CBC natural e sem pré-tratamento e o efeito da sua adição na resistência à compressão e absorção de água de tijolos de solo-cimento. Este estudo 
compõe uma importante etapa na promoção do aproveitamento e aplicação da CBC em materiais de construção no Vale do São Patrício, Goiás, visto que a presença deste resíduo na região é abundante.

\section{Materiais e métodos}

\section{Materiais}

Foram utilizados como matéria prima para a fabricação dos tijolos de solo-cimento silte argiloso (solo) coletado em jazida na cidade de Rialma (Goiás, Brasil), cimento Portland CP II-E-32 e cinza residual da queima do bagaço da cana-de-açúcar fornecida pela CRV - Industrial LTDA, Unidade Carmo do Rio Verde, Goiás.

\section{Caracterização e classificação do solo}

$\mathrm{O}$ solo foi coletado à $50 \mathrm{~cm}$ de profundidade, de modo que não houvesse matéria orgânica, em seguida transportado, e destorroado. As amostras coletadas foram preparadas seguindo-se as recomendações descritas na NBR 6457 (ABNT, 2016a). O solo coletado foi classificado como silte argiloso, de acordo com a textura, através do Triângulo Textural, utilizando os teores de areia, silte e argila, de acordo com o Manual Técnico de Pedologia (INSTITUTO..., 2007). A massa específica dos sólidos do solo foi determinada no Laboratório de Solos, do Instituto Federal Goiano - Campus Ceres conforme apresentado nos procedimentos da Figura 1 e de acordo com o Manual de Métodos de Análise de Solos (BRASIL, 1997).

A análise granulométrica dos sólidos foi realizada de acordo com a norma NBR 7181 (ABNT, 2016b). A determinação do índice de plasticidade e dos limites de consistência do solo foi realizada através dos procedimentos descritos na NBR 7180 (ABNT, 2016c). O limite de liquidez foi determinado segundo a NBR 6459 (ABNT, 2016d). Inicialmente, a amostra previamente preparada foi inserida em uma cápsula de porcelana, adicionando-se água em pequenas quantidades, amassando e revolvendo com o auxílio de uma espátula até a obtenção de uma pasta homogênea. A massa plástica foi transferida para a concha do aparelho de Casagrande e moldada com espessura na parte central de $1 \mathrm{~cm}$ e em seguida executado o ensaio.

\section{Caracterização química da cinza de bagaço de cana-de-açúcar}

As cinzas do bagaço de cana-de-açúcar (CBC) foram secas em estufa durante 3 horas a $110^{\circ} \mathrm{C}$ para remoção da umidade presente. Após a secagem, o resíduo foi moído manualmente a seco, e em seguida peneirado em peneira de $300 \mu \mathrm{m}$. A CBC foi caracterizada quimicamente segundo o Documento 236 - Procedimentos para Análise Lignocelulósica da EMBRAPA (MORAIS et al., 2010). Para a determinação do teor de umidade a amostra teve sua massa medida em um recipiente inerte, em seguida, o conjunto material-recipiente foi levado à estufa onde permaneceu secando durante $3 \mathrm{~h}$ a $105{ }^{\circ} \mathrm{C}$, e em seguida novamente pesado. Para a análise do teor percentual de cinzas o conjunto material-recipiente foi aquecido em forno mufla com rampa de aquecimento de $9,6^{\circ} \mathrm{C} / \mathrm{min}$ entre temperatura ambiente e $600{ }^{\circ} \mathrm{C}$, onde permaneceu em isoterma durante três horas. Ao final desse tempo, a temperatura foi reduzida para $200{ }^{\circ} \mathrm{C} \mathrm{em}$ uma hora. $\mathrm{O}$ conjunto cadinhoamostra foi removido, resfriado em dessecador por 30 minutos e em seguida massa de cinzas foi medida.

A análise do teor de extrativos presentes na $\mathrm{CBC}$ foi realizada com auxílio de um cartucho de amostra adicionado a um extrator de Soxhlet. Inicialmente, foi adicionado solvente a um balão de vidro, e conectado ao extrator. O conjunto balão-extrator foi colocado em uma manta aquecedora e conectado ao condensador. A temperatura foi ajustada de modo que o número de refluxos ocorridos não fosse menor que 24 no período de 5 h de extração. Após o procedimento, o balão foi removido e o extrato transferido para um recipiente de pesagem, previamente seco e com a massa determinada, e inserido em uma estufa durante $1 \mathrm{~h} \mathrm{a} 105^{\circ} \mathrm{C}$. Em seguida, o material foi removido da estufa, transferido para dessecador até atingir temperatura ambiente, e então medida a massa final.

A composição elementar da CBC foi determinada via combustão em analisador elementar (Perkin Elmer 2400 Series II). Na análise foram medidos os teores em porcentagem de carbono (C), hidrogênio (H) e nitrogênio $(\mathrm{N})$ presentes na amostra de $\mathrm{CBC}$. A análise mineralógica foi realizada por meio da técnica de difração de raio X (difratômetro SHIMADZU - DRX-6000), usando radiação monocromática de $\mathrm{Cu}-\mathrm{K} \alpha$, com uma varredura de $10^{\circ}$ até $80^{\circ}(2 \theta)$ e velocidade do escaneamento de $2 \% \mathrm{~min}$. 
Figura 1 - Análise textual pelo método do densímetro: adição de solução de $\mathrm{NaOH}$ ao material (a) e inserção do densímetro para realização da leitura de silte+argila (b)

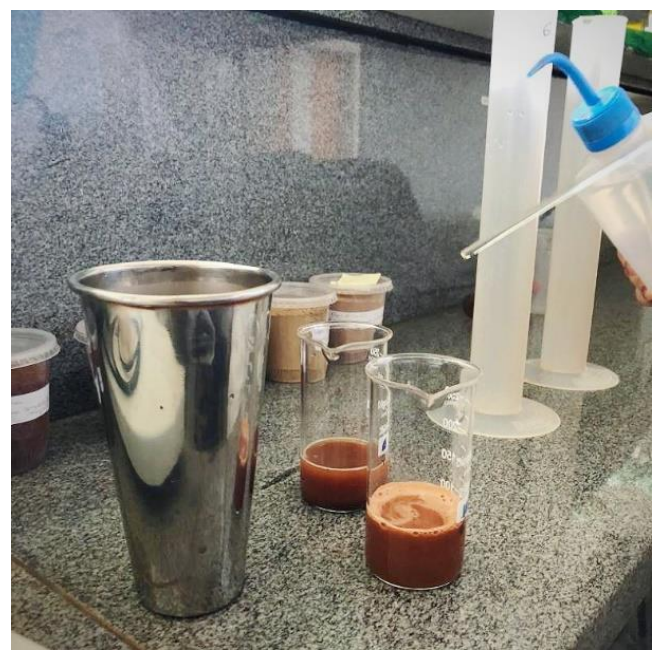

(a)

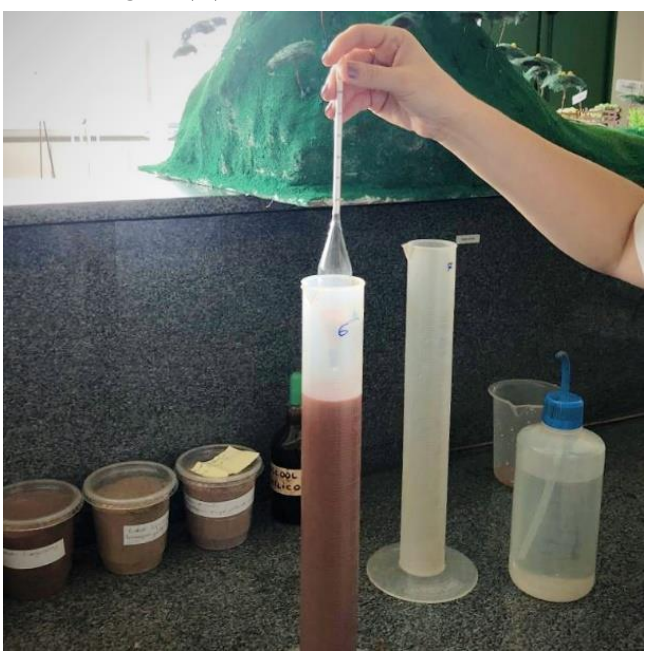

(b)

\section{Moldagem e cura dos corpos de prova}

A mistura de solo-cimento de referência foi submetida a ensaio de compactação realizado conforme estabelece a norma NBR 12023 (ABNT, 2012a), onde a relação água-cimento definida e utilizada em todos os traços foi de $16 \%(\mathrm{~m} / \mathrm{m})$. A mistura de solo-cimento utilizada foi definida em 1:11 de acordo com as recomendações do Boletim Técnico - Fabricação de Tijolos de Solo-Cimento com a Utilização de Prensas Manuais (ASSOCIAÇÃO..., 2000). A incorporação da cinza do bagaço de cana a mistura foi realizada variando os teores de cimento em relação a cinza, assim, foram utilizadas as proporções (cimento/CBC), em massa, de 100/0, 90/10, 80/20 e 50/50. Assim, foi adotada a nomenclatura solo-cimento (cimento/CBC) para descrever os diferentes grupos, sendo eles: Solo puro, solo-cimento (100/0), solo-cimento (90/10), solocimento $(80 / 20)$ e solo-cimento (50/50).

Os corpos de prova (CPs) cilíndricos de solo-cimento foram moldados de acordo com a norma NBR 12024 (ABNT, 2012). A moldagem foi realizada compactando-se o material em um molde cilíndrico de 12,7 x 10 $\mathrm{cm}$. Neste procedimento, o material foi compactado em 3 camadas com 26 golpes cada, utilizando um soquete de $2,5 \mathrm{~kg}$ liberado de uma altura de $30 \mathrm{~cm}$. Foram moldados quatro CPs para cada grupo conforme apresentado na Figura 2. Após a moldagem, os CPs permaneceram curando em temperatura ambiente e umidade controlada durante 28 dias.

\section{Absorção de água e caracterização mecânica}

A análise de absorção de água e caracterização mecânica dos corpos de prova foi realizada, em um conjunto de quatro CPs de cada grupo, seguindo os procedimentos estabelecidos pela norma NBR 8492 (ABNT, 2012c). Inicialmente, os CPs foram secos em estufa à $105{ }^{\circ} \mathrm{C}$ até a constância da massa e pesados. Em seguida, foram imersos em água durante $24 \mathrm{~h}$, retirados e novamente pesados para a obtenção da capacidade de absorção.

As propriedades mecânicas foram avaliadas através do ensaio de resistência à compressão simples realizado em uma Máquina Universal de Ensaios Mecânicos CONTECO (I-3058) com uma célula de carga de 100T e velocidade do ensaio de $200 \mathrm{Kgf} / \mathrm{min}$. Os resultados do ensaio foram interpretados com o auxílio de análise de variância ANOVA. Quando se observaram diferenças significativas, os materiais foram comparados entre si, por meio do teste de Tukey com $5 \%$ de probabilidade.

\section{Resultados e discussão}

\section{Caracterização do solo e distribuição do tamanho das partículas}

As curvas de distribuição do tamanho das partículas, obtida via peneiramento, são apresentadas na Figura 3. Os resultados apontam que as amostras compõem diferentes faixas de tamanhos, onde o cimento apresenta 
as menores partículas registradas. A análise granulométrica do resíduo de CBC in natura mostra que o material apresenta um alto percentual de partículas grosseiras relacionadas as longas fibras de bagaço não queimado. Essas partículas porosas podem ser resultado da combustão de matéria orgânica durante a queima do bagaço (AMARAL, 2014). As partículas esponjosas absorvem a água usada para a reação do cimento, sendo necessário a moagem, já que a reação pozolânica pode ser altamente ativada quando o tamanho da partícula é pequeno (KIATTIKOMOL et al., 2001).

O solo coletado apresentou composição de 35,70\% areia, 37,50\% argila e 26,90\% silte de acordo com a análise granulométrica dos sólidos. A curva do solo aponta um maior valor do coeficiente de uniformidade, indicando que a massa de areia/argila/silte consiste em diferentes faixas de tamanhos de partículas. A distribuição de matérias-primas usadas é muito importante, pois afeta diretamente a porosidade total dos corpos de prova (ELERT; CULTRONE, 2003). Em relação a granulometria do solo, foi possível observar que o mesmo atende as especificações da NBR 10832 (ABNT, 1989) no qual exige que o solo tenha uma porcentagem passante de $100 \%$ na peneira de $4,8 \mathrm{~mm}$ e 10 a $50 \%$ na peneira de $0,075 \mathrm{~mm}$. O solo estudado possui uma grande fração de argila e por isso o cimento tem papel importante em sua estabilização, diminuindo a plasticidade da fração argilosa, podendo ser ou não acompanhada de aumento na resistência mecânica (DIAS, 2012).

Os limites de consistência foram influenciados consideravelmente pela textura do solo. As frações de argila e silte apresentaram relação positiva sobre os valores, sendo os Limites de Liquidez (LL) e de Plasticidade (LP) registrados de 54,30\% e 33,60\% respectivamente e um Índice de Plasticidade (IP) igual à $20,60 \%$. Segundo as recomendações da NBR 10832 (ABNT, 1989), o solo utilizado para a fabricação de tijolos de solo-cimento deve apresentar limite de liquidez e limite de plasticidade menor ou igual a $45 \%$ e $18 \%$ respectivamente. Os resultados da análise do solo estudado apresentam valores inferiores ao recomendado, sendo altamente plástico de acordo com os limites de Atterberg e apresentando partículas que aderem firmemente umas às outras.

A análise da consistência do solo permitiu compreender sobre a sua suscetibilidade à compactação, o que exige um manejo adequado em relação a umidade. Quanto maior o índice de plasticidade do solo, maior será a dificuldade em estabilizá-lo, uma vez que o material estará mais sujeito às variações dimensionais, provenientes do seu inchamento quando úmido e da sua retração quando seco (MIELI, 2009). O solo foi classificado como silte argiloso a partir da análise do triângulo textural, de acordo com o Manual Técnico de Pedologia do IBGE (INSTITUTO..., 2007) e demonstrou impróprio para a produção do tijolo solo-cimento, o que exigiu maiores quantidades de aglomerante para o processo de estabilização.

\section{Figura 2 - Corpos de prova cilíndricos com dimensões de 12,7 x $10 \mathrm{~cm}$}

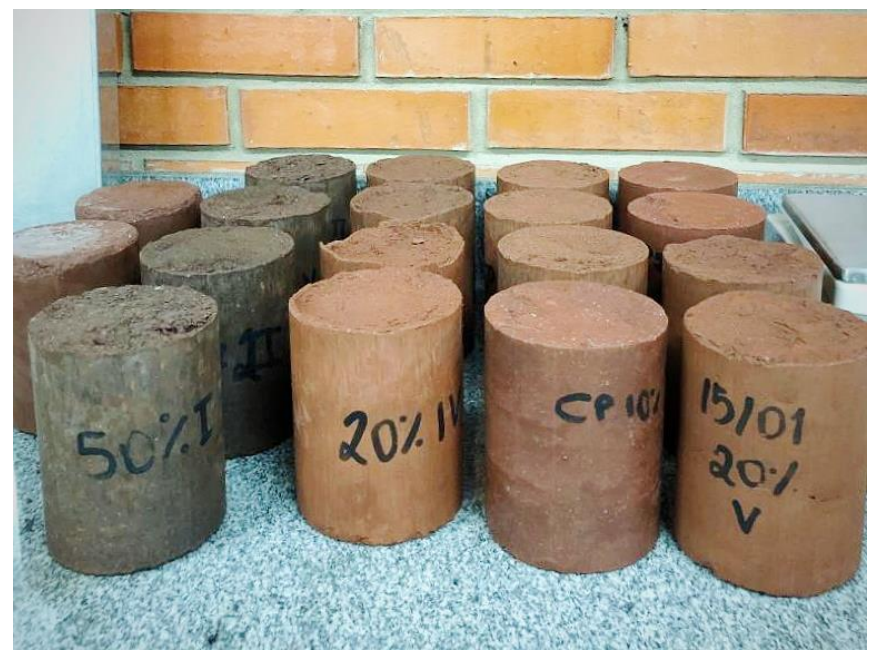


Figura 3 - Distribuição do tamanho das partículas do solo, $\mathrm{CBC}$ in natura, $\mathrm{CBC}$ moída e cimento

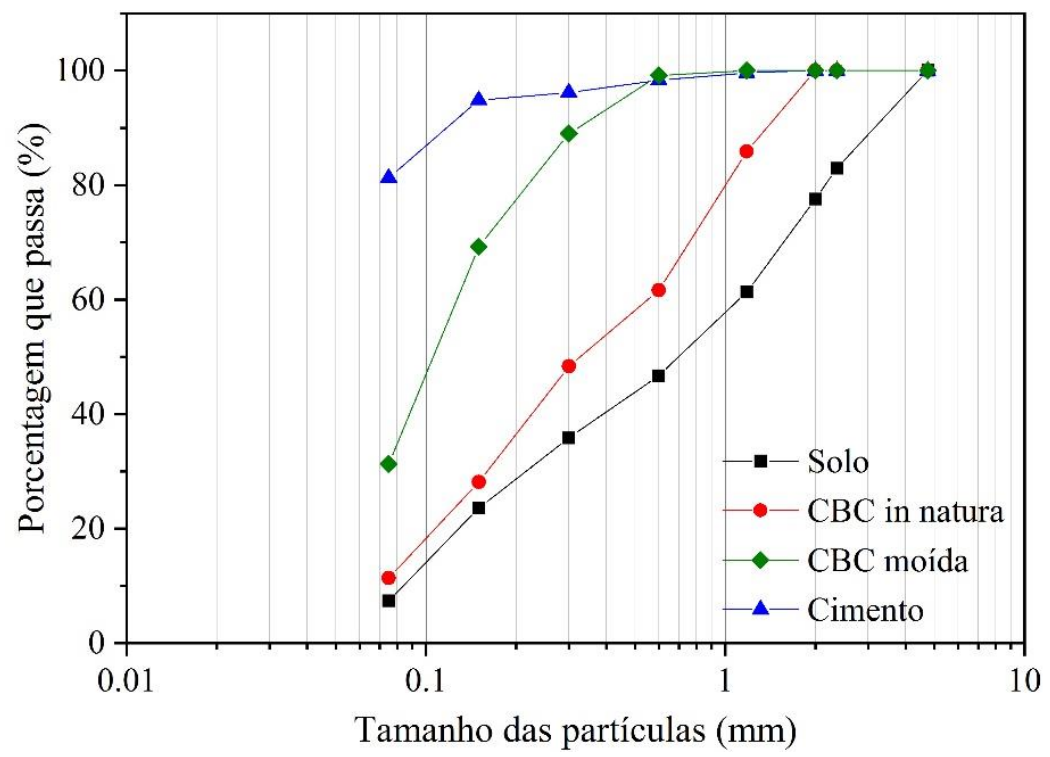

\section{Caracterização química da cinza de bagaço de cana-de-açúcar}

O bagaço de cana consiste em aproximadamente 50\% de celulose, $25 \%$ de hemiceluloses e $25 \%$ de lignina (MAHESH et al., 2017). Após a queima, o resíduo do bagaço de cana-de-açúcar quando analisado in natura apresentou umidade de 50,2\%. A quantidade de cinzas presente na amostra foi de 47,1\%, indicando a presença de minerais, assim como resíduos de subprodutos usados durante sua produção. O teor de extrativos da CBC foi de 5,24\%. Segundo os resultados da análise elementar CHN, o teor de carbono presente na cinza foi de $5,48 \%$, este percentual está relacionado às condições de combustão da biomassa. O teor de carbono é responsável pela cor preta das cinzas e é provavelmente o resultado de condições de combustão descontroladas (KATARE; MADUWAR, 2017). A concentração de nitrogênio presente na amostra foi de 0,09\%, semelhante aos encontrados por Arif, Clark e Lake (2016). O processamento da biomassa, a concentração de carbono, a composição mineralógica, e tamanho das partículas, são algumas das principais características que influenciam na reatividade da cinza (CORDEIRO; TOLEDO FILHO; FAIRBAIRN, 2009).

A Figura 4 apresenta o difratograma de raios-X (DRX) da CBC. Através da análise dos picos foi possível identificar as seguintes fases cristalinas na amostra: quartzo, cristobalita e hematita. A presença de quartzo e cristobalita indicam biomassa queimada a alta temperatura, o que está de acordo com os resultados obtidos por Arif, Clark e Lake (2016). A grande porcentagem de quartzo na CBC deve-se à presença de areia, cerca de 2,0\% em peso, aderida à cana-de-açúcar durante o processo de colheita (XU et al., 2019). A presença de quartzo na cinza pode reduzir a reatividade da pozolana.

A cristobalita indica a fase cristalina da sílica. A cristalização ocorreu devido a elevada temperatura de combustão no processo de queima (KATARE; MADUWAR, 2017). Para melhorar as propriedades pozolânicas é necessário a transformação da sílica cristalina em sílica amorfa, que é mais reativa (FREITAS, 2005). Quando a CBC possui um teor de sílica cristalina relativamente alto na forma de quartzo, ela ainda pode ser usada em materiais de construção como substituto ou preenchedor de areia (SALES; LIMA, 2010). Devido à estrutura cristalina, diversos estudos indicam a utilização de CBC como substituto parcial do cimento Portland e de agregados miúdos (ARIF; CLARK; KAKE, 2016; MAHESH et al., 2017; FARIA et al., 2012).

\section{Resistência mecânica e absorção de água}

Os resultados dos ensaios de resistência à compressão foram obtidos através dos rompimentos dos corpos de prova $(\mathrm{CP})$ aos 28 dias e são apresentados na Tabela 1. Todos os grupos que possuíam cimento em sua composição obtiveram valores de resistência aceitáveis aos 28 dias de cura.

O tijolo de solo puro apresentou resistência mecânica inferior a todos os grupos. Em relação aos corpos de prova de solo-cimento, a resistência diminuiu de acordo com o aumento da substituição parcial do cimento 
pela CBC, o que está de acordo com resultados encontrados por Kazmi et al. (2016). Este resultado está associado a redução da quantidade de cimento no traço, visto que o cimento é o responsável por promover o aumento de resistência da mistura (AMARAL, 2014). A Figura 5 apresenta a região de fratura dos corpos de prova após o rompimento no ensaio de compressão simples.

Figura 4 - Padrão de DRX da CBC

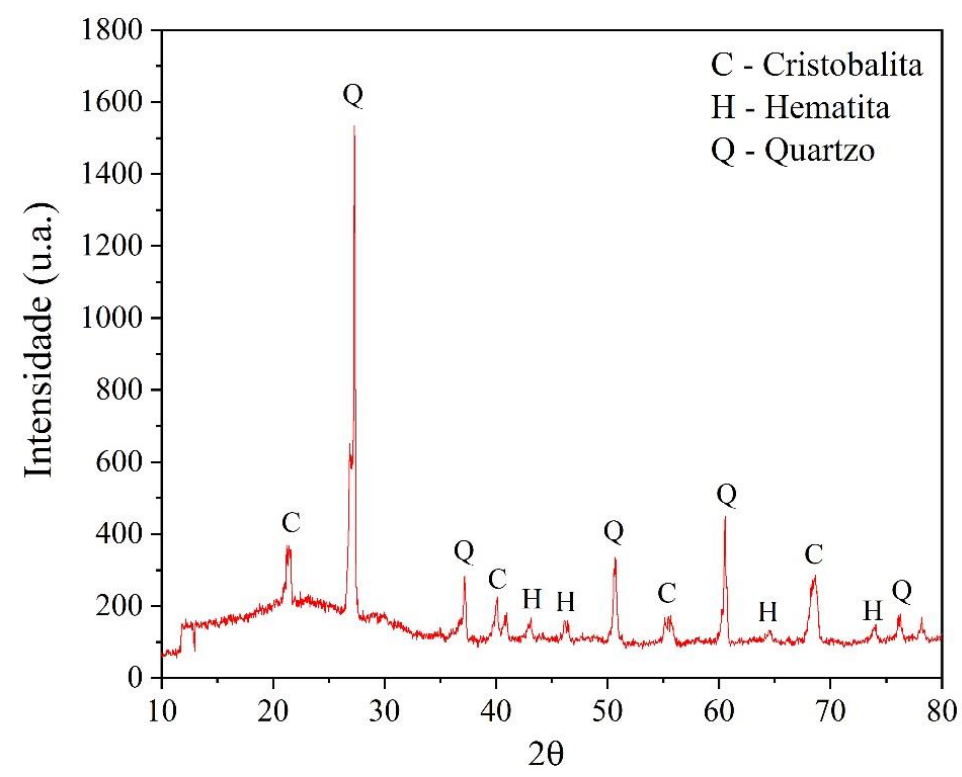

Tabela 1 - Resistência à compressão média dos corpos de prova

\begin{tabular}{c|c|c|c}
\hline Mistura & $\begin{array}{c}\text { Cimento } \\
(\boldsymbol{\%})\end{array}$ & $\begin{array}{c}\text { CBC } \\
(\boldsymbol{\%})\end{array}$ & $\begin{array}{c}\text { Limite de } \\
\text { resistência (MPa) }\end{array}$ \\
\hline Solo & 0 & 0 & $1,15 \pm 0,53$ \\
Solo-cimento (100/0) & 100 & 0 & $4,05 \pm 0,72$ \\
Solo-cimento (90/10) & 90 & 10 & $3,45 \pm 0,70$ \\
Solo-cimento (80/20) & 80 & 20 & $3,73 \pm 0,56$ \\
Solo-cimento (50/50) & 50 & 50 & $2,35 \pm 0,39$ \\
\hline
\end{tabular}

Figura 5 - Fratura dos corpos de prova após ensaio de compressão: solo-cimento (80/20) (a) e solocimento $(100 / 0)(b)$

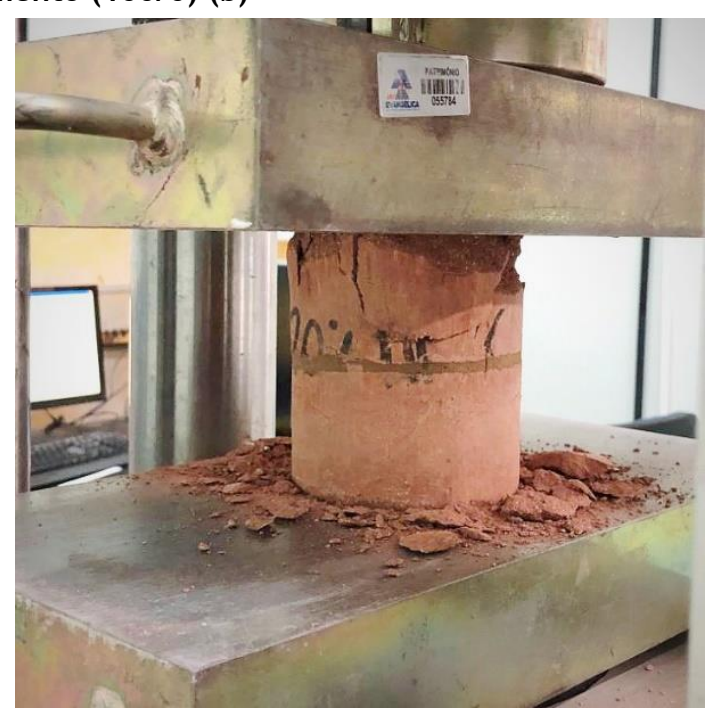

(a)

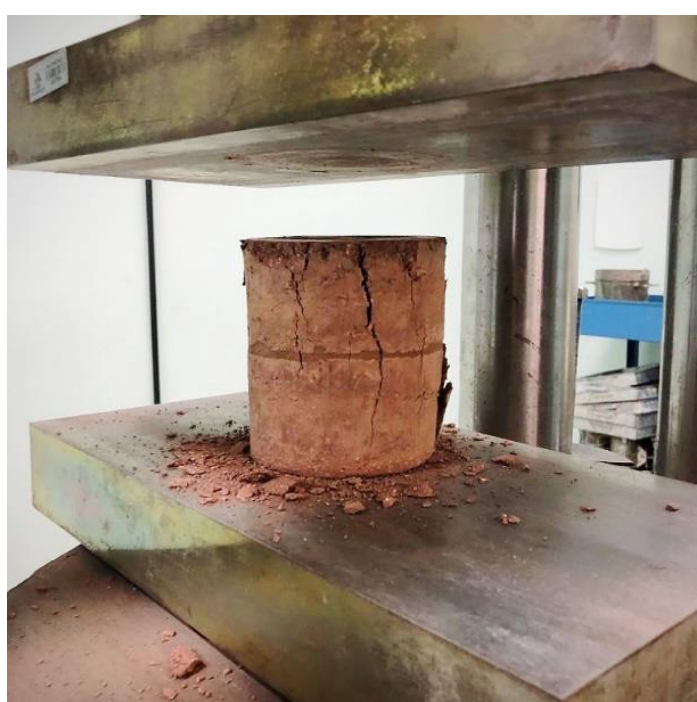

(b) 
O gráfico da Figura 6 apresenta uma comparação entre todos os grupos de tijolos de acordo com a composição de cada traço. É possível observar que todos os grupos, com exceção do solo puro, apresentaram valores da resistência média superior das recomendações da NBR 8492 para tijolo maciço de solo-cimento (2,0 MPa) (ABNT, 2012c). A manutenção da resistência mecânica dos corpos de prova com adição da CBC acontece devido o teor de pozolana e seu efeito de enchimento ao ser inserido na composição solo-cimento (ARIF; CLARK; LAKE, 2016). Entre os grupos estudados que utilizaram CBC em substituição parcial do cimento, o solo-cimento (80/20) apresentou a maior resistência mecânica. Segundo Ganesan et al. (2007), a substituição parcial do cimento em $20 \%$ apresenta os melhores resultados de resistência mecânica em materiais cimentícios (GANESAN; RAJAGOPAL; THANGAVEL, 2007).

A Figura 7 apresenta o resultado da análise de Tukey com nível de significância de $p=0,05$. No gráfico, o intervalo de valores em vermelho aponta os tijolos que são significativamente diferentes entre si, caso o intervalo intercepte o valor 0 os tijolos são significativamente iguais. De acordo com a análise ANOVA, o valor da resistência à compressão é significativamente equivalente quando comparado os tijolos solocimento (100/0) em relação aos tijolos solo-cimento (90/10) e solo-cimento (80/20).

A análise indica que os grupos solo-cimento (100/0) e solo-cimento (80/20) são estatisticamente iguais em um nível de $95 \%$ de confiança. Apesar de ter apresentado a menor resistência média entre os grupos de solocimento o teste Tukey apontou que o tijolo solo-cimento (50/50) exibe resistência mecânica estatisticamente semelhante ao solo-cimento (90/10), indicando que o uso desta composição pode substituir os grupos que utilizaram a substituição do cimento em até 40\%. Segundo Jamsawang et al. (2017) a substituição parcial de $20 \%$ do cimento pela CBC é considera como quantidade ideal, visto que apresenta o mesmo efeito fortalecedor se comparado ao cimento usado isoladamente. Entretanto, a adição gradativa da CBC na composição do tijolo pode comprometer a resistência mecânica se adicionada em grandes quantidades (KAZMI et al., 2016). Isso explica a perda de resistência entre o grupo solo-cimento (50/50) se comparado com os demais tijolos.

A Tabela 2 apresenta os resultados referentes à análise da absorção de água estudada a partir de uma média aritmética de 4 repetições.

Nos corpos de prova é possível observar uma diminuição da capacidade de absorção de água ao longo da substituição do cimento pela CBC. O ensaio de absorção de água não foi aplicado aos tijolos de solo. O traço utilizado para produção do tijolo solo-cimento é satisfatório em relação a absorção de água, visto que os resultados obtidos são inferiores ao valor médio de 20\% estabelecido pela NBR 8492 (ABNT, 2012c). As partículas finas da CBC têm efeito físico de preencher os microporos do solo permitindo a manutenção da resistência mecânica e o aumento da relação água cimento (ALMEIDA et al., 2015). O aumento da adição de cinza nos corpos de prova reduziu a porosidade interna dos tijolos levando-os a uma menor capacidade de absorção de água.

Figura 6 - Resistência à compressão aos 28 dias de cura dos tijolos de solo-cimento

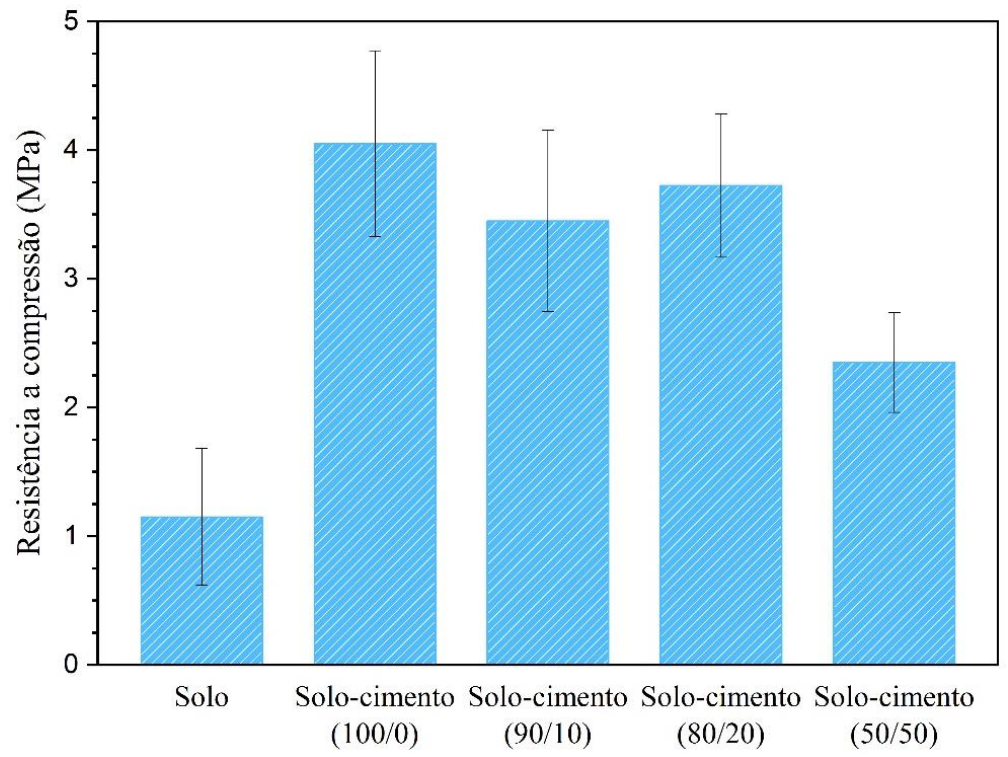

76 Moura, E. M. de; Sales, J. N. B. de; Nascimento, N. C. do; Sousa, V. M. Z. de; Costa e Silva, D. D.; Libera Junior, V. D. 
Figura 7 - Gráficos da análise de Tukey para a limite da resistência dos tijolos de solo-cimento

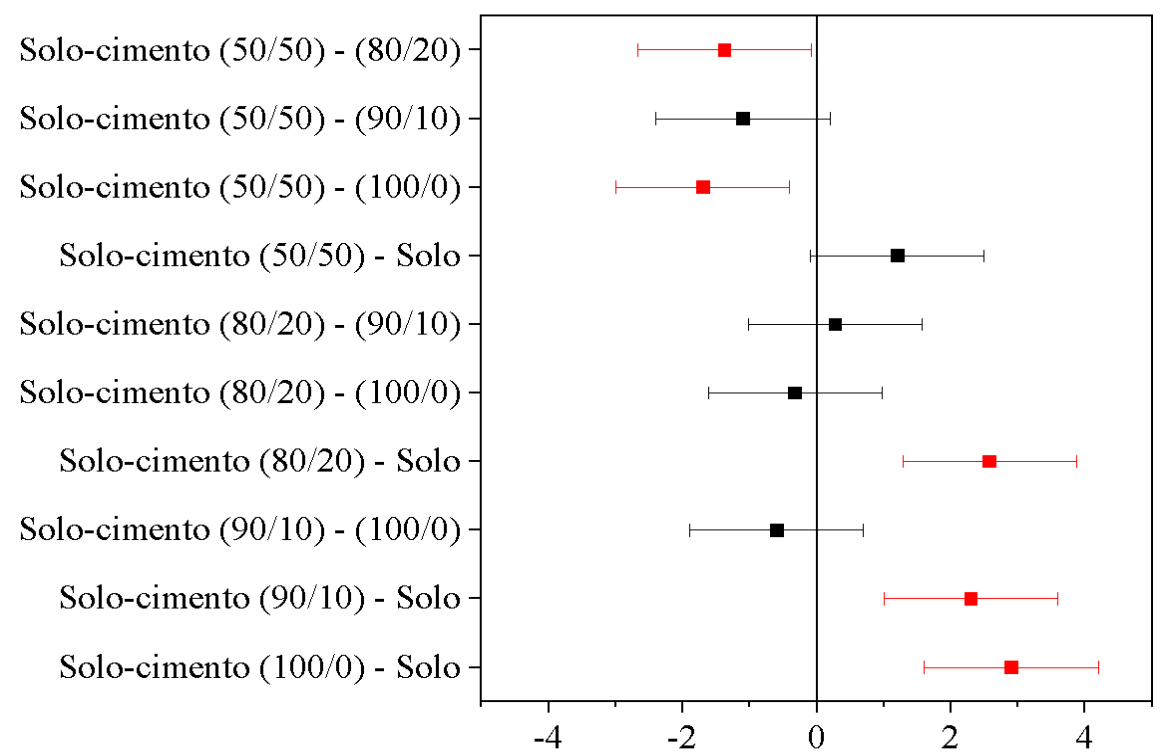

Tabela 2 - Média das porcentagens de absorção de água

\begin{tabular}{c|c|c|c}
\hline Mistura & $\begin{array}{c}\text { Cimento } \\
(\boldsymbol{\%})\end{array}$ & $\begin{array}{c}\text { CBC } \\
(\boldsymbol{\%})\end{array}$ & $\begin{array}{c}\text { Absorção de } \\
\text { água (\%) }\end{array}$ \\
\hline Solo & 0 & 0 & - \\
Solo-cimento (100/0) & 100 & 0 & $9,65 \pm 1,35$ \\
Solo-cimento (90/10) & 90 & 10 & $9,44 \pm 1,96$ \\
Solo-cimento (80/20) & 80 & 20 & $7,46 \pm 0,32$ \\
Solo-cimento (50/50) & 50 & 50 & $3,61 \pm 0,92$ \\
\hline
\end{tabular}

\section{Conclusões}

A avaliação dos efeitos da adição de cinza do bagaço de cana-de-açúcar no tijolo de solo-cimento foi possível graças a variação de composições estudadas. Neste estudo a relação cimento/cinza foi selecionada e as interações entre as proporções sugeridas apresentaram níveis de significância distintos. A análise granulométrica do solo indicou uma porcentagem passante de $100 \%$ na peneira de $4,8 \mathrm{~mm}$ e 10 a $50 \%$ na peneira de 0,075 mm, o que está de acordo com a norma vigente. A análise da CBC através da técnica de difração de raios-X comprovou a presença de quartzo e cristobalita na cinza, o que indica que a biomassa foi queimada a alta temperatura. Apesar da apresentação de sílica na forma cristalina, é aceitável a utilização da CBC como substituto parcial do cimento Portland e de agregados miúdos. Após a adição de cinza e cimento os corpos de prova de solo-cimento mais que dobraram sua resistência à compressão em relação ao tijolo de solo puro. Entre os grupos que utilizaram cinza como substituto do cimento, a substituição parcial em até $20 \%$ do cimento pela CBC não alterou significativamente as propriedades mecânicas dos tijolos de solocimento. A manutenção da resistência dos corpos de prova (CP) com adição de cinza do bagaço de cana-deaçúcar acontece, devido ao teor de pozolana e efeito de enchimento da composição solo-cimento. Após produzidos, todos os corpos de prova apresentaram capacidade de absorção menor que 20\%. Apesar de apresentar um baixo estado de reatividade, a CBC se provou como um subproduto viável para aplicação como material cimentício suplementar em componentes de construção, visto que, seu teor de sílica na forma de quartzo é um dos principais elementos componentes na areia natural. Apesar das conclusões animadoras, a avaliação de outras propriedades físicas dos tijolos de solo-cimento é fundamental para amparar a aplicação do material produzido em situações práticas.

\section{Referências}

ALMEIDA, F. C. R. et al. Sugarcane bagasse ash sand (SBAS): Brazilian agroindustrial by-product for use in mortar. Construction and Building Materials, v. 82, p. 31-38, 2015. 
AMARAL, M. C. Avaliação da incorporação de resíduo de cinzas de bagaço de cana-de-açúcar em tijolo solo-cimento. Rio de Janeiro, 2014. 98 f. Dissertação (Mestrado em Engenharia e Ciência dos Materiais) - Universidade Estadual do Norte Fluminense, Campos dos Goytacazes, 2014.

ARIF, E.; CLARK, M. W.; LAKE, N. Sugar cane bagasse ash from a high efficiency co-generation boiler: Applications in cement and mortar production. Construction and Building Materials, v. 128, p. 287-297, 2016.

ASSOCIAÇÃO BRASILEIRA DE CIMENTO PORTLAND. Fabricação de tijolos de solo-cimento com a utilização de prensas manuais. 3. ed. rev. atual. São Paulo: ABCP, 2000.

ASSOCIAÇÃO BRASILEIRA DE NORMAS TÉCNICAS. NBR 10832: fabricação de tijolo maciço de solo-cimento com utilização de prensa manual. Rio de Janeiro, 1989.

ASSOCIAÇÃO BRASILEIRA DE NORMAS TÉCNICAS. NBR 12023: solo-cimento: ensaio de compactação. Rio de Janeiro, 2012a.

ASSOCIAÇÃO BRASILEIRA DE NORMAS TÉCNICAS. NBR 12024: solo-cimento: moldagem e cura de corpos de prova cilíndricos: procedimento. Rio de Janeiro, 2012b.

ASSOCIAÇÃO BRASILEIRA DE NORMAS TÉCNICAS. NBR 6457: amostras de solo: preparação para ensaios de compactação e ensaios de caracterização. Rio de Janeiro, 2016a.

ASSOCIAÇÃO BRASILEIRA DE NORMAS TÉCNICAS. NBR 6459: solo: determinação do limite de liquidez. Rio de Janeiro, 2016d.

ASSOCIAÇÃO BRASILEIRA DE NORMAS TÉCNICAS. NBR 7180: solo: determinação do limite de plasticidade. Rio de Janeiro, 2016c.

ASSOCIAÇÃO BRASILEIRA DE NORMAS TÉCNICAS. NBR 7181: solo: análise granulométrica. Rio de Janeiro, 2016b.

ASSOCIAÇÃO BRASILEIRA DE NORMAS TÉCNICAS. NBR 8492: tijolo de solo-cimento: análise dimensional, determinação da resistência à compressão e da absorção de água: Método de ensaio. Rio de Janeiro, 2012c.

BRASIL. Ministério da Agricultura e do Abastecimento. Centro Nacional de Pesquisa de Solos. Manual de métodos de análise de solo. 2. ed. Rio de Janeiro: EMBRAPA, 1997.

CHANDRA PAUL, S. et al. Agricultural solid waste as source of supplementary cementitious materials in developing countries. Materials, v. 12, p. 1112, 2019.

CORDEIRO, G. C.; TOLEDO FILHO, R. D.; FAIRBAIRN, E. M. R. Effect of calcination temperature on the pozzolanic activity of sugar cane bagasse ash. Construction and Building Materials, v. 23, p. 33013303, 2009.

DANSO, H. et al. Physical, mechanical and durability properties of soil building blocks reinforced with natural fibres. Construction and Building Materials, v. 101, p. 797-809, 2015.

DIAS, J. J. F. M. S. Tratamento de solos com cimento para obtenção de melhores características mecânicas. Lisboa, 2012. 117 f. Dissertação (Mestrado em Engenharia Civil) - Universidade Nova de Lisboa, Lisboa, 2012.

ELERT, K.; CULTRONE, G. Durability of bricks used in the conservation of historic buildings-influence of composition and microstructure. Jornal of Cultural Heritage, v. 4, n. 2, p. 91-99, 2003.

FARIA, K. C. P.; GURGEL, R. F.; HOLANDA, J. N. F. Recycling of sugarcane bagasse ash waste in the production of clay bricks. Journal of Environmental Management, v. 101, p. 7-12, 2012.

FREITAS, E. S. Caracterização de cinza de bagaço da Cana-de-açúcar do município de Campos dos Goytacazes para uso na construção civil. Rio de Janeiro, 2005. 97 f. Dissertação (Mestrado em Engenharia Civil) - Universidade Estadual do Norte Fluminense, Campos dos Goytacazes, 2005.

FRÍAS, M.; VILLAR, E.; SAVASTANO, H. Brazilian sugar cane bagasse ashes from the cogeneration industry as active pozzolans for cement manufacture. Cement and Concrete Composites, v. 33, p. 490496, 2011.

GANESAN, K.; RAJAGOPAL, K.; THANGAVEL, K. Evaluation of bagasse ash as supplementary cementitious material. Construction and Building Materials, v. 29, p. 515-524, 2007. 
INSTITUTO BRASILEIRO DE GEOGRAFIA E ESTATÍSTICA. Manual Técnico de Pedologia. 2. ed. Rio de Janeiro, 2007.

JAMSAWANG, P. et al. Improvement of soft clay with cement and bagasse ash waste. Construction and Building Materials, v. 154, n. 12, p. 61-71, 2017.

KANTIRANIS, N. Recycling of sugar-ash: a raw feed material for rotary kilns. Waste Manage, v. 24, p. 999-1004, 2004.

KATARE, V. D.; MADUWAR, M. V. Experimental characterization of sugarcane biomass ash: a review. Construction and Building Materials, v. 152, p. 1-15, 2017.

KAZMI, S. M. S. et al. Manufacturing of sustainable clay bricks: Utilization of waste sugarcane bagasse and rice husk ashes. Construction and Building Materials, v. 120, p. 29-41, 2016.

KIATTIKOMOL, K. et al. A study of ground coarse fly ashes with different finenesses from various sources as pozzolanic materials. Cement and Concrete Composites, v. 23, n. 4/5, p. 335-343, 2001.

KOLLING, E. M.; TROGELLO, E.; MODOLO, A. J. Avaliação da resistência mecânica de diferentes traços de solo-cimento estabilizados com areia. Revista Eletrônica de Materiais e Processos, v. 7, n. 3, p. 185191, 2012.

MAHESH, B. et al. Usage of sugarcane bagasse ash in concrete. International Journal of Engineering Research and General Science, v. 5, p. 218-224, 2017.

MARTIRENA, J. F. et al. Use of waste of sugar industry as pozzolana in lime-pozzolana binders: study of reaction. Cement and Concrete Research, v. 28, p. 1525-1536, 1998.

MIELI, P. H. Avaliação do tijolo modular de solo-cimento como material na construção civil. Rio de Janeiro, 2009. 48 f. TCC (Graduação) - Curso de Engenharia de Materiais, Departamento de Engenharia Metalúrgica e de Materiais, Universidade Federal do Rio de Janeiro, Rio de Janeiro, 2009.

OLIVEIRA, M. P. et al. Estudo do caulim calcinado como material de substituição parcial do cimento Portland. In: CONFERÊNCIA BRASILEIRA DE MATERIAIS E TECNOLOGIAS NÃO CONVENCIONAIS: HABITAÇÕES E INFRA-ESTRUTURA DE INTERESSE SOCIAL BRASIL, Pirassununga, 2004. Anais [...] São Paulo, 2004.

RODRIGUES, L. P.; HOLANDA, J. N. F. Recycling of water treatment plant waste for production of soilcement bricks. Procedia Materials Science, v. 8, p. 197-202, 2015.

SALES, A.; LIMA, S. A. Use of Brazilian sugarcane bagasse ash in concrete as sand replacement. Waste Manage, v. 30, p. 1114-1122, 2010.

SCHNEIDER, M. et al. Sustainable cement production: present and future. Cement and Concret Research, v. 41, p. 642-650, 2011.

TRAN, K. Q.; SATOMI, T.; TAKAHASHI, H. Improvement of mechanical behavior of cemented soil reinforced with waste cornsilk fibers. Construction and Building Materials, v. 178, p. 204-210, 2018.

VALENCIANO, M. C. M.; FREIRE, W. J. Características físicas e mecânicas de misturas de solo, cimento e cinzas de bagaço de cana-de-açúcar. Engenharia Agrícola, v. 24, n. 3, p. 484-492, 2004.

$\mathrm{XU}, \mathrm{Q}$. et al. Characteristics and applications of sugar cane bagasse ash waste in cementitious materials.

Materials, v. 12, n. 1, p. 39, 2019. 


\title{
Elda Marra de Moura
}

Departamento de Engenharia Civil, Centro Universitário de Anápolis | UniEVANGÉLICA | Av. Brasil, Qd 13 Setor Morada Verde | Ceres - GO - Brasil | CEP 76300-000 | Tel.: (62) 99202-6252 | E-mail: eldamarram@gmail.com

\section{Joel Neto Bispo de Sales}

Departamento de Engenharia Civil, Centro Universitário de Anápolis | UniEVANGÉLICA | Tel.: (62) 99294-1329 | E-mail: netojoel_engcivil@hotmail.com

\section{Natalia Cristina do Nascimento}

Departamento de Engenharia Civil, Centro Universitário de Anápolis | UniEVANGÉLICA | Tel.:(62) 99860-6140 | E-mail: ncn.nataliacristina@gmail.com

\section{Vitor Magalini Zago de Sousa}

Departamento de Engenharia Civil, Centro Universitário de Anápolis | UniEVANGÉLICA | Tel.: (62) 99902-4989 | E-mail: vitormagalinizago@gmail.com

\section{Danilo Duarte Costa e Silva}

Escola de Engenharia Civil e Ambiental | Universidade Federal de Goiás | Av. Universitária, Quadra 86, Lote Área 1488, Setor Leste Universitário | Goiânia - GO - Brasil | CEP 74605-220 | Tel.: (62) 99161-9999 | E-mail: daniloduarte777@yahoo.com.br

\section{Vilson Dalla Libera Junior}

Departamento de Engenharia Civil, Centro Universitário de Anápolis | UniEVANGÉLICA | Tel.: (62) 99310-0446 | E-mail: vilsondalla@hotmail.com

\author{
Ambiente Construído \\ Revista da Associação Nacional de Tecnologia do Ambiente Construído \\ Av. Osvaldo Aranha, $99-3^{\circ}$ andar, Centro \\ Porto Alegre - RS - Brasil \\ CEP 90035-190 \\ Telefone: +55 (51) 3308-4084 \\ Fax: +55 (51) 3308-4054 \\ www.seer.ufrgs.br/ambienteconstruido \\ E-mail: ambienteconstruido@ufrgs.br
}

(c) (i)

80 Moura, E. M. de; Sales, J. N. B. de; Nascimento, N. C. do; Sousa, V. M. Z. de; Costa e Silva, D. D.; Libera Junior, V. D. 